\title{
Gadd45 $\beta$ expression in chondrosarcoma: A pilot study for diagnostic and biological implications in histological grading
}

\author{
Michihisa Zenmyo', Akihide Tanimoto², Harutoshi Sakakima³, Masahiro Yokouchi', Satoshi Nagano', \\ Takuya Yamamoto', Yasuhiro Ishido', Setsuro Komiya ${ }^{1}$, Kosei ljiri ${ }^{*}$
}

\begin{abstract}
Background: Although the diagnosis of chondrosarcoma, especially the distinction between enchondroma and low-grade chondrosarcoma or low-grade chondrosarcoma and high-grade chondrosarcoma, is pathologically difficult, differential diagnosis is very important because the treatment strategies for these diseases are completely different. The grading system is crucial in predicting biologic behavior and prognosis, however, exact pathological grading is difficult using only routine examinations because the criteria of the grading system are not necessarily definitive. Growth arrest and DNA damage-inducible protein $45 \beta$ (GADD45 $\beta$ ) is an essential molecule for chondrocytes during terminal differentiation. In the present study, we investigated the immunohistochemical expression of GADD45 $\beta$ in enchondroma, and chondrosarcoma of histological grades I, II, and III, to clarify the diagnostic significance of GADD45 $\beta$ in pathological grading of chondrosarcoma.
\end{abstract}

Methods: Twenty samples (enchondroma $=6$, chondrosarcoma grade I $=7$, grade $\|=6$, grade $I I=1$ ) were used for immunohistochemical analysis to investigate the expression of GADD45 $\beta$. Quantitative analysis was performed to compare the number of GADD45 $\beta$ positive cells and pathological grading.

Results: Over $70 \%$ of the cells in enchondromas expressed GADD45 $\beta$. On the other hand, the expression of GADD45 $\beta$ decreased significantly according to the histological grade of chondrosarcoma (grade I: $45 \%$; grade II: 13.8\%; and grade III: $3.8 \%$ ).

Conclusions: The association of GADD45 $\beta$ expression and pathological grading of chondrosarcoma in the present study suggests that the immunohistochemical study of GADD45 $\beta$ may be a specific diagnostic parameter for chondrosarcoma cell differentiation.

\section{Background}

Chondrosarcoma is the second most frequent primary malignant bone tumor $[1,2]$. Because of its recalcitrance to chemotherapy and radiotherapy, chondrosarcoma is primarily treated with surgery, and the clinical prognosis of chondrosarcoma has been correlated with the grading of the histological malignancy [3].

For pathological consideration, distinguishing benign (enchondroma) from low-grade chondrosarcoma, or lowgrade chondrosarcoma from high-grade chondrosarcoma,

\footnotetext{
* Correspondence: kosei2@m.kufm.kagoshima-u.ac.jp

'Orthopaedic Surgery, Graduate School of Medical and Dental Sciences,

Kagoshima University, Kagoshima, Japan

Full list of author information is available at the end of the article
}

is one of the most frequent diagnostic dilemmas facing orthopedic oncologists.

Enchondroma is a very common and benign cartilaginous tissue tumor that occurs within bones. Approximately $69 \%$ of the patients are in the first and second decades of life [4]. More than $49 \%$ of the tumors are in the small bones of the hands and feet, particularly in the phalanges. Unlike chondrosarcoma, enchondroma rarely develops in the pelvis or ribs. The pathological distinction between enchondroma and low-grade chondrosarcoma is, however, not always easy because of their similar cytology and cellularity. Chondrosarcoma has a broad array of presentations in pathology and clinical course. Chondrosarcoma is primarily a tumor of adulthood and old age. 
Approximately $62 \%$ of the patients are in the fourth to sixth decades. More than two-thirds of the tumors are in the trunk, including the pelvis, ribs, and shoulder girdle. The pathological grading of chondrosarcoma is based on cellularity, nuclear atypia, and pleomorphism [5]; however, in some borderline cases, exact histological grading is difficult using only routine histopathological examinations because the criteria of the grading system are not necessarily definitive [6]. Therefore, correlative interpretation of histopathological, imaging, and clinical information is currently used for making this distinction.

Several authors have reported supplementary methods, including the evaluation of DNA synthesis and content [7,8], flow cytometry [9], p53 [10], MIB-1 [11], COX-2 [6], and p21 [3], to assess the prognosis of patients with chondrosarcoma. These methods are, however, based on non-specific phenomena in chondrocytic differentiation.

Chondrogenesis, i.e., cartilage formation including chondrocyte differentiation and maturation, is a process that occurs during skeletal development. This process occurs in stages beginning with mesenchymal cell recruitment and migration, proliferation, and condensation, followed by chondroprogenitor cell determination and differentiation. Finally, chondrocyte differentiation is terminated by hypertrophy. Bone morphogenetic proteins (BMPs), which were originally identified as molecules that induce ectopic endochondral ossification [12], set the stage for bone morphogenesis by initiating chondroprogenitor cell determination and differentiation and regulate the later stages of chondrocyte maturation and hypertrophic phenotype [13].

We previously reported the growth arrest and DNA damage-inducible protein $45 \beta$ (GADD $45 \beta$ ) as an early responding gene to $\mathrm{BMP}-2$ stimulation in the chondrocyte cell line [14]. The expression of GADD45 $\beta$ gradually increased along with chondrocyte differentiation from the proliferation phase to hypertrophic phase. GADD $45 \beta$ stimulates MMP-13 (a marker of terminal differentiation of hypertrophic chondrocytes) promoter activity in chondrocytes through the JNK-mediated phosphorylation of JunD, partnered with Fra2 and in synergy with Runx2. These facts suggested that GADD $45 \beta$ plays an essential role during chondrocyte terminal differentiation.

In the present study, we investigated the immunohistochemical expression of GADD $45 \beta$ in enchondroma and chondrosarcoma of histological grades I, II, and III, to clarify the diagnostic significance of GADD $45 \beta$ in histological grading of chondrosarcoma.

\section{Methods}

\section{Study samples}

Formalin-fixed, paraffin-embedded tissues collected between 1978 and 2009 were obtained from the
Department of Pathology, Graduate School of Medical and Dental Sciences, Kagoshima University upon approval of the ethics committee. The characteristics of the patients are summarized in Table 1.

\section{Pathological review and grading}

Histological slides of the tumors from all the patients were reviewed by 2 or 3 pathologists. The histological diagnosis was based on the textbook definition [15]. The histological grade of the chondrosarcomas was determined on the basis of the nuclear size, nuclear staining (hyperchromasia), and cellularity according to the World Health Organization Classification of Bone Tumors (2002) [15].

\section{Immunohistochemical analysis}

The immunohistochemical analysis was performed using the labeled streptavidin-biotin method using goat polyclonal anti-human GADD45 $\beta$ (C-18; 1:1000) antibody (Santa Cruz Biotechnology, Inc. CA, USA). The results were evaluated by 2 investigators, who were unaware of the pathological grading of the samples. We calculated the proportion of stained tumor cells after performing nuclear staining.

Quantitative analysis of the GADD $45 \beta$ immunoreactive cells was performed. The immunostained sections were photographed at $100 \times$ magnification with a microscope, and the GADD $45 \beta$ immunoreactive cells and non-positive cells were counted in 4 fields. We

\section{Table 1 Patient characteristics}

\begin{tabular}{|c|c|c|c|c|}
\hline Case & $\begin{array}{l}\text { Sex/ } \\
\text { Age }\end{array}$ & Location & $\begin{array}{c}\text { Pathology \& } \\
\text { Grade }\end{array}$ & $\begin{array}{c}\text { GADD45b positivity } \\
\text { (\%) }\end{array}$ \\
\hline 1 & $0^{n} / 43$ & Femur & Enchondroma & 68.9 \\
\hline 2 & $0^{n} / 16$ & Metatarsal & Enchondroma & 81.6 \\
\hline 3 & @/46 & Phalanx & Enchondroma & 74.4 \\
\hline 4 & $0 / 32$ & Phalanx & Enchondroma & 83.5 \\
\hline 5 & \$/58 & Femur & Enchondroma & 66.6 \\
\hline 6 & Q/54 & Phalanx & Enchondroma & 68.8 \\
\hline 7 & $\$ / 52$ & Femur & CSA grade I & 67.3 \\
\hline 8 & $07 / 39$ & Pelvis & CSA grade I & 55.5 \\
\hline 9 & O/46 & Femur & CSA grade I & 44.6 \\
\hline 10 & $0^{n} / 61$ & Femur & CSA grade I & 49.2 \\
\hline 11 & $\sigma^{x} / 18$ & Tibia & CSA grade I & 29.8 \\
\hline 12 & $\sigma^{2} / 67$ & Mandible & CSA grade I & 38.1 \\
\hline 13 & Q/32 & Pelvis & CSA grade I & 31.0 \\
\hline 14 & Q/50 & Femur & CSA grade $\|$ & 11.8 \\
\hline 15 & @/64 & Rib & CSA grade II & 3.9 \\
\hline 16 & Q/72 & Femur & CSA grade $\|$ & 14.8 \\
\hline 17 & Q/55 & Pelvis & CSA grade $\|$ & 5.9 \\
\hline 18 & $\sigma^{2} / 44$ & Femur & CSA grade $\|$ & 11.9 \\
\hline 19 & $\sigma^{2} / 67$ & Mandible & CSA grade II & 34.0 \\
\hline 20 & $\sigma^{2} / 61$ & Mandible & CSA grade III & 3.8 \\
\hline
\end{tabular}




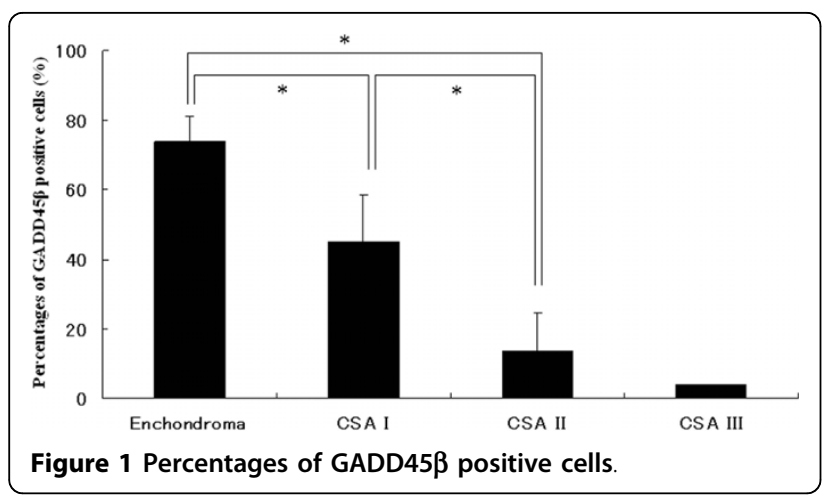

calculated the percentages of GADD45 $\beta$ positive cells. Statistical comparisons in each grade (enchondroma, grade I and II) were performed by a one-way ANOVA using Stat-View version 5.0. If significance was achieved, a post-hoc Fisher's protected least significant differences (PLSD) test was performed to determine whether significant differences existed. Significance was set at $\mathrm{p}<0.05$.

\section{Results}

The patient data and lesion characteristics are listed in Table 1. There were 6 cases of enchondroma and 14 of chondrosarcoma, including 7 with grade I, 6 with grade
II, and 1 with grade III. Enchondromas, on average, showed $74 \%$ reactivity for GADD $45 \beta$ (68.9-83.5\%). In the case of chondrosarcomas, the percentage of GADD $45 \beta$ positive cells was inversely proportional to the tumor grade. GADD $45 \beta$ was expressed in $45 \%$ (29.8-67.3\%) of the cells in grade I chondrosarcoma and $13.8 \%(1.2-34.1 \%)$ of cells in grade II. There were statistically significant differences among the three groups except grade III chondrosarcoma. (Fig. 1)

In grade II chondrosarcomas, comparatively mature cells, which had plump nuclei and were surrounded by cartilage matrix, were positive for GADD $45 \beta$. In contrast, most of the atypical spindle cells, which had enlarged hyperchromatic nuclei and myxomatous matrix, were negative. (Fig. 2C) Although we had only 1 case of grade III chondrosarcoma, only $3.8 \%$ of the cells were positive for GADD45 $\beta$.

\section{Discussion}

The pathological grade of chondrosarcomas is considered to be the most useful predictor of the clinical outcome and course of the treatment for these tumors. While criteria for pathological grade of chondrosarcomas have been published, the application of these criteria is difficult and requires expert judgement [16].

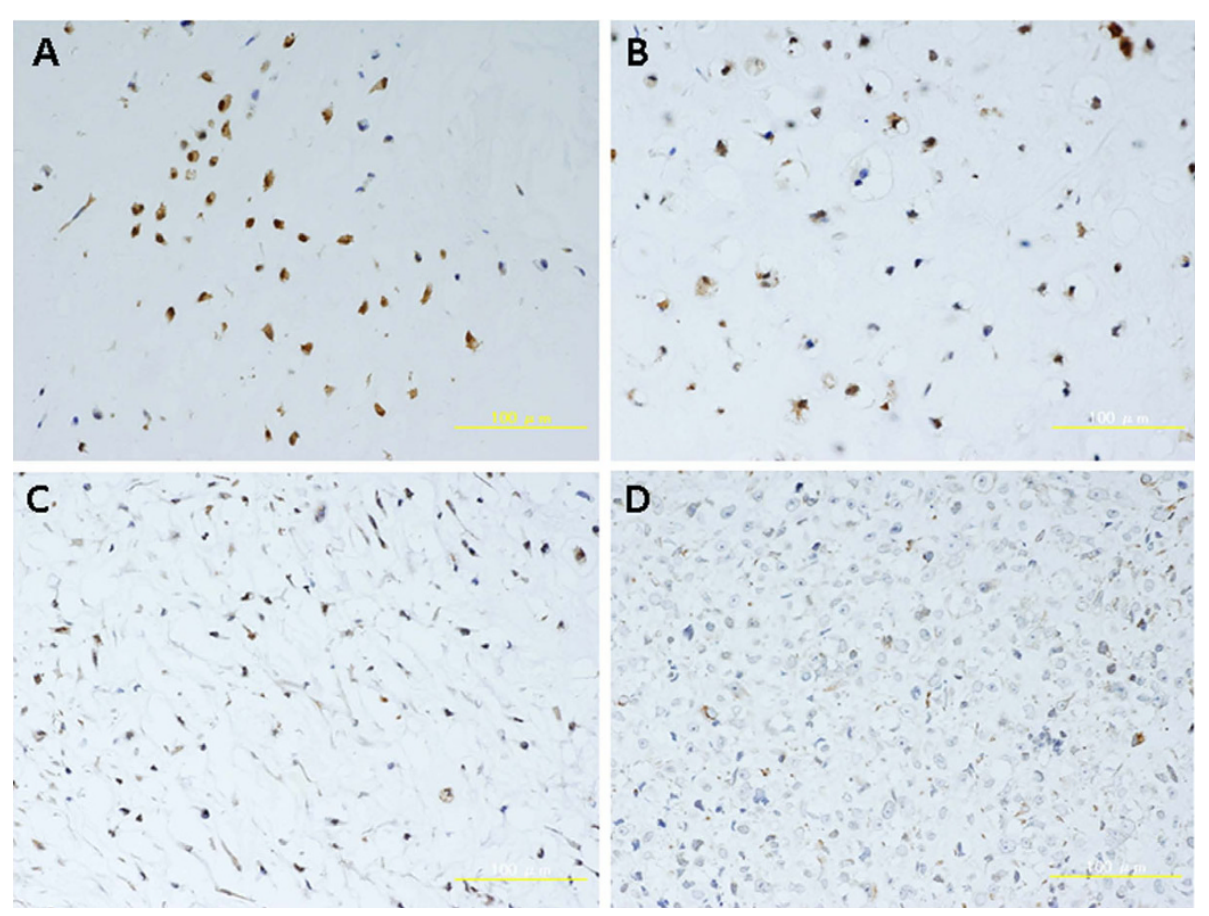

Figure 2 Immunohistochemical staining for GADD45 $\beta$ A) Enchondroma, B) Grade I Chondrosarcoma, C) Grade II Chondrosarcoma, D) Grade III Chondrosarcoma. Chondrocytes which have plump nuclei and are surrounded by cartilage matrix, are positive for GADD45 $\beta$. In contrast, most of the atypical and immature cells in grade II or III chondrosarcoma are negative. Over $70 \%$ of the cells in enchondromas expressed GADD45 $\beta$. In chondrosarcomas, the rate of GADD45 $\beta$ positive cells was inversely proportional to its grade. There were statistically significant differences among three groups except grade III chondrosarcoma. (*: P < 0.01). 
Therefore, correlative interpretation of histopathological, imaging, and clinical information is currently used as a method to provide the appropriate prognostic information. Recently, the reliability of histopathological and radiological grading of cartilaginous neoplasms in long bones was studied by 9 recognized musculoskeletal pathologists and 8 recognized musculoskeletal radiologists [17]. They reviewed 49 consecutive cases of cartilaginous lesions in long bones and estimated the interobserver reliability for the grading of cartilaginous neoplasms in long bones to be 0.443 for pathologists and 0.345 for radiologists. These data demonstrate that the interpretation of histopathological features from cartilaginous lesions has some limits. These data encouraged the efforts to develop better prospective pathological interpretation algorithms or better prospective imaging protocols.

The present study provides the first immunohistochemical evidence that GADD $45 \beta$ expression is significantly associated with the grading of chondrosarcomas. GADD45 $\beta$ is a member of the GADD45 family of small $(18 \mathrm{kDa})$ proteins, which are known to be associated with cell growth control, apoptotic cell death, and the cellular response to DNA damage [18]. Initially, GADD $45 \beta$ encoded by MyD118 was identified as a myeloid differentiation primary response gene activated by IL-6 in murine myeloid leukemia cells upon induction of terminal differentiation [19].

Formation of the vertebral skeleton through endochondral ossification is a physiological process, which involves progressive differentiation of proliferating chondrocytes to growth-arrested hypertrophic chondrocytes. Interestingly, the GADD $45 \beta$ protein localized prominently in the nucleus of the late-stage hypertrophic chondrocytes, but not of the proliferating chondrocytes. Although GADD $45 \beta$ is an abundant protein in chondrocytes, overexpression of GADD $45 \beta$ in terminally differentiated chondrocytes in the growth plate promotes JNK and p38 signaling cascades to regulate both MMP-13 and Col10a1 expression, which are crucial for the maintenance of the terminal differentiated phenotype [20]. Therefore, the association of GADD $45 \beta$ expression and pathological grading of chondrosarcoma in the present study suggests that immunohistochemical study of GADD $45 \beta$ may be a specific diagnostic parameter for chondrosarcoma cell differentiation.

In this study, there was overlap in the proportion of GADD $45 \beta$-expressing cells between enchondroma and grade I chondrosarcoma (CSA), as shown for Case 7, and between grade I CSA and grade II CSA, as shown for Case 19. The limitation of this pilot study of GADD45 $\beta$ expression in chondrogenic tumors was the small number of subjects. Therefore, the study should be conducted on a larger number of subjects to determine whether the number of such "overlap" cases would increase.

\section{Conclusions}

In conclusion, our data suggests that immunohistochemical study of GADD45 $\beta$ provides valuable diagnostic information and facilitates examination of histological grading. More studies involving larger number of patients must be performed to further characterize its potential diagnostic validity.

\section{Acknowledgements}

This research was supported by KAKENHI-20591787(KI).

\section{Author details}

'Orthopaedic Surgery, Graduate School of Medical and Dental Sciences, Kagoshima University, Kagoshima, Japan. ${ }^{2}$ Molecular and Cellular Pathology, Graduate School of Medical and Dental Sciences, Kagoshima University, Kagoshima, Japan. ${ }^{3}$ School of Health Sciences, Faculty of Medicine, Kagoshima University, Kagoshima, Japan.

\section{Authors' contributions}

ZM, AT, MY, SN carried out the immunohistochemical studies. TY, YI participated in the design of the study, and $\mathrm{SH}$ contributed in the statistical analysis. SK and $\mathrm{KI}$ conceived the study and drafted the manuscript. All authors read and approved the final manuscript.

\section{Competing interests}

The authors declare that they have no competing interests.

Received: 30 August 2010 Accepted: 13 October 2010

Published: 13 October 2010

\section{References}

1. Larsson SE, Lorentzon R: The incidence of malignant primary bone tumours in relation to age, sex and site. A study of osteogenic sarcoma, chondrosarcoma, and Ewing's sarcoma diagnosed in Sweden from 1958 to 1968. J Bone joint Surg Br 1974, 56:534-540.

2. Hartley AL, Blair V, Harris M, Birch JM, Banerjee SS, Freemont AJ, McClure J, McWilliam LJ: Sarcomas in north west England:II. Incidence. $\mathrm{Br} J$ Cancer 1991, 64:1145-1150.

3. Hiraoka K, Zenmyo M, Komiya S, Kawabata R, Yokouchi M, Suzuki R, Hamada T, Kato S, Nagata K: Relationship of p21(waf1/cip1) and differentiation on chondrosarcoma cells. Virchows Arch 2002, 440:285-290.

4. Unni KK: General aspects and data on 11,087 cases. In Dahlin's bone tumor Edited by: Lippincott-Raven, Minnesota, Fifth 1996, 25.

5. Bertoni HD, Czerniak B: Malignant cartilage tumor. Bone tumors (Mo): Mosby, St Louis 1998, 353-440.

6. Endo M, Matsumura T, Yamaguchi T: Cyclooxygenase-2 overexpression associated with a poor prognosis in chondrosarcomas. Human pathology 2006, 37:471-476.

7. Alho A, Skjeldal S, Melvik JE, Pettersen EO, Larsen TE: The clinical importance of DNA synthesis and aneuploidy in bone and soft tissue tumours. Anticancer Res 1993, 13:2383-2387.

8. Kreicbergs A, Boquist L, Borssén B, Larsson SE: Prognostic factors in chondrosarcoma: a comparative study of cellular DNA content and clinicopathologic features. Cancer 1982, 50:577-583.

9. Alho A, Connor JF, Mankin HJ, Schiller AL, Campbell CJ: Assessment of malignancy of cartilage tumors using flow cytometry. A preliminary report. J Bone Joint Surg Am 1983, 65:779-785.

10. Dobashi $Y$, Sugimura H, Sato A, Hirabayashi T, Kanda H, Kitagawa T, Kawaguchi N, Imamura T, Machinami R: Possible association of p53 overexpression and mutation with high-grade chondrosarcoma. Diagn Mol Pathol 1993, 2:257-263.

11. Nawa G, Ueda T, Mori S, Yoshikawa H, Fukuda H, Ishiguro S, Funai H, Uchida A: Prognostic significance of Ki67(MIB-1) proliferation index and p53 over-expression in chondrosarcomas. Int J Cancer 1996, 69:86-9. 
12. Urist MR: Bone: Formation by autoinduction. Science 1965, 150:893-899.

13. Goldring MB, Tsuchimochi K, ljiri $K$ : The control of chondrogenesis. J Cellular Biochem 2006, 97:33-44.

14. Ijiri K, Zerbini LF, Peng H, Correa RG, Lu B, Walsh N, Zhao Y, Taniguchi N, Huang XL, Otu H, Wang H, Wang JF, Komiya S, Ducy P, Rahman MU, Flavell RA, Gravallese EM, Oettgen P, Libermann TA, Goldring MB: A novel role for Gadd $45 b$ as a mediator of MMp-13 gene expression during chondrocyte terminal differentiation. J Biol Chem 2005, 280:38544-38554.

15. Bertoni F, Bacchini P, Hogendoorn PCW: Chondrosarcoma. In World health Organization Classification of Tumours. Pathology and genetics of tumours of soft tissue and bone. Edited by: Fletcher CDM, Unni KK, Mertens F. IARC Press, Lyon; 2002:247-251.

16. Murphey MD, Flemming DJ, Boyea SR, Bojescul JA, Sweet DE, Temple HT: Enchondroma versus chondrosarcoma in the appendicular skeleton: differentiating features. Radiographics 1998, 18:1213-1237.

17. Skeletal lesions interobserver correlation among expert diagnosticians study group: Reliability of histopathological and radiologic grading of cartilaginous neoplasms in long bones. J Bone joint Surg Am 2007, 89:2113-2123.

18. Amanullah A, Azam N, Balliet A, Hollander C, Hoffman B, Fornace A, Liebermann D: Cell signalling: cell survival and a Gadd45-factor deficiency. Nature 2003, 424:741-742.

19. Selvakumaran M, Lin HK, Sjin RT, Reed JC, Liebermann DA, Hoffman B: The novel primary response gene MyD118 and the proto-oncogenes myb, myc, and bcl-2 modulate transforming growth factor beta 1-induced apoptosis of myeloid leukemia cells. Mol Cell Biol 1994, 14:2352-2360.

20. Tsuchimochi $K$, Otero $M$, Dragomir CL, Plumb DA, Zerbini LF, Libermann TA Marcu KB, Komiya S, Jjiri K, Goldring MB: Gadd45beta enhances Col10a1 transcription via the MTK1/MKK3/6/p38 axis and activation of C/ EBPbeta-TAD4 in terminally differentiating chondrocytes. J Biol Chem 2010, 285:8395-83407.

doi:10.1186/1746-1596-5-69

Cite this article as: Zenmyo et al:: Gadd45 $\beta$ expression in chondrosarcoma: A pilot study for diagnostic and biological implications in histological grading. Diagnostic Pathology 2010 5:69.

\section{Submit your next manuscript to BioMed Central and take full advantage of:}

- Convenient online submission

- Thorough peer review

- No space constraints or color figure charges

- Immediate publication on acceptance

- Inclusion in PubMed, CAS, Scopus and Google Scholar

- Research which is freely available for redistribution

Submit your manuscript at www.biomedcentral.com/submit
Ciomed Central 